\title{
Comparing the effect of gums on the growth of lactobacillus species in laboratory medium and fluid milk
}

\begin{abstract}
This study compared the growth of three Lactobacillus strains in the presence of gums during a 12 hour incubation period at $37^{\circ} \mathrm{C}$ in order to determine which gum promoted the most growth. Our results showed that the populations of Lactobacillus rhamnosus GGB101 and Lactobacillus rhamnosus GGB103 were significantly higher in milk compared to growth in a laboratory medium, whereas Lactobacillus reuteri DSM20016 performed better in the medium. The recommended level of log $6 \mathrm{CFU}$ g- 1 was exceeded for all tested trains during the incubation period. The addition of xanthan led to the highest growth of L. rhamnosus GGB101 $(8.81 \pm 0.01 \log \mathrm{CFU} / \mathrm{mL})$ and L. rhamnosus GGB103 $(8.32 \pm 0.01 \log \mathrm{CFU} / \mathrm{mL})$ in milk. Carrageenan-maltodextrin promoted the highest growth $(8.30 \pm 0.23 \log \mathrm{CFU} / \mathrm{mL})$ of $L$. reuteri DSM 20016 in the medium and was found to support significant growth of Lactobacillus strains in both milk and medium. Our results showed that carrageenanmaltodextrin, xanthan and carrageenan could thus serve as functional ingredients for the enhanced growth and viability of Lactobacillus strains to promote quality probiotic dairy foods and thereby promote human health.
\end{abstract}

Keywords:gums, Lactobacillus, milk, basal medium, probiotics, prebiotics
Volume 2 Issue 3 - 2015

\author{
Bernice Dzifa Karlton-Senaye, ${ }^{1,2}$ Leonard \\ Lamont Williams, ${ }^{2}$ Salam Adnan Ibrahim' \\ 'Center for Excellence in Post-Harvest Technologies, USA \\ ${ }^{2}$ North Carolina Agricultural and Technical State University, USA
}

Correspondence: Bernice Dzifa Karlton-Senaye, Center for Excellence in Post Harvest Technologies, The North Carolina Research Campus, UNC Nutrition Research Building, 500 Laureate Way, Kannapolis, NC 2808I, USA, Office (704) 2505709, Fax (704) 2505709, Email bekarlton@gmail.com

Received: May 15, 2015 | Published: July 25, 2015
Abbreviations: CFU, colony forming unit; MRS, deman rogosa and sharpe; ANOVA, analysis of variance

\section{Introduction}

Probiotics are living microorganisms (mainly Lactobacillus and Bifidobacteria), which confer health benefits to the host when taken in adequate amounts. ${ }^{1}$ Most strains of Lactobacillus and Bifidobacteria are known for their probiotic qualities with associated health benefits., ${ }^{2,3}$ Currently, Lactobacilli with probiotics are added to a variety of functional foods, and several studies have demonstrated their beneficial properties in human and animal health. These health benefits include alleviation of lactose intolerance, acceleration of intestinal mobility, reinforcement of gut mucosal immunity, decreased risk associated with mutagenicity and carcinogenicity, hypocholesterolemic effects, reduced duration of diarrhea, prevention of inflammatory bowel disease, prevention of colon cancer, prevention of allergies, and treatment and inhibition of intestinal pathogens. ${ }^{4}$ However, such health benefits can only be attained when the probiotic Lactobacillus strains are viable and exceed a population of six million. ${ }^{4}$ These healthpromoting microorganisms are vulnerable to various environmental stressors to which they are subjected during fermentation, storage, and digestion. The survival of Lactobacillus microorganisms is also affected by food composition and the interaction between different bacterial strains. ${ }^{5}$ To attain the target population level of six million, $100 \mathrm{~g}$ of food products containing $10^{6}-10^{7}$ live cells must be ingested daily. ${ }^{6}$

Prebiotics are defined as non-digestible food substances that selectively stimulate the growth and activity of limited gut microorganisms and promote health benefits. ${ }^{7}$ Some prebiotic carbohydrates such as fructooligosaccharides, lactulose, inulin and galactooligosaccharides from lactose (GOS-La) are currently available in the market. ${ }^{6.8}$ However, there is also considerable interest in learning more about new carbohydrates with potential prebioticqualities. Polysaccharides gums (such as, carrageenan, pectin, xanthan, alginate, gellan, zedo, konjac, starch, cellulose, and chitosan), proteins (casein, whey, gelatin, $\beta$-lacto globulin) and wax have been studied as prebiotics that could improve the viability of probiotic microorganisms. ${ }^{9-12}$

Gums are complex polysaccharides extracted from plant, animal and microbial sources. ${ }^{5}$ Gums impact sensory qualities, contribute fiber to foods ${ }^{13}$ and promote growth in probiotics. ${ }^{12}$ Gums are also used as thickeners and binders in cosmetics, medications, inks, paint, paper, and adhesives. ${ }^{13}$ Gums are comprised of food ingredients such as carbohydrates, sugars, salts and minerals that could serve as additional carbon sources to enhance the growth of probiotic organisms in food and in the human gut.

Thus, the aim of this study was to investigate the effect of different gums on the growth of three Lactobacillus strains in a laboratory medium and fluid milk as a practical means to enhance the viability of probiotics and ultimately improve the quality of dairy products.

\section{Materials and methods}

\section{Culture activation}

Pure cultures of L. reuteri DSM20016, L. reuteri SD2112, L. rhamnosus GG B101, and L. rhamnosus GG B103 strains were obtained from the stock culture collection of the Food Microbiology and Biotechnology Laboratory at North Carolina Agricultural 
\&Technical State University (Greensboro, NC, USA). Bacterial cultures were activated by transferring $100 \mu$ l of stock culture to $10 \mathrm{~mL}$ of deMan Rogosa Sharpe (MRS) broth (Neogen, Lansing, MI) and incubating at $37^{\circ} \mathrm{C}$ for $24 \mathrm{~h}$.

\section{Gum composition, preparation and inoculation}

Fluid milk (1\% milk fat) and a modified basal medium were prepared by gradually dissolving $0.5 \%(\mathrm{w} / \mathrm{v})$ of each of the following gums: pectin (PE), carrageenan (CA), carrageenan-maltodextrin $(\mathrm{MC})$, pectin-carrageenan $(\mathrm{PC})$, locust bean (LB), guar (GU), inulin (IN) and guar-locust bean-carrageenan (GL) and xanthan (XA) (Maryland, GA, USA) to a $100 \mathrm{~mL}$ batch of $1 \%$ fat liquid milk at $70^{\circ} \mathrm{C}$.
Samples without gums served as a negative control. Milk and media samples were then treated in a water bath at $65^{\circ} \mathrm{C}$ for $30 \mathrm{~min}$ and cooled to $42^{\circ} \mathrm{C}$ before use.

\section{Preparation and composition of basal medium}

A basal medium was prepared by dissolving the following: $2 \mathrm{~g}$ Proteose peptone, $2.0 \mathrm{~g}$ yeast extract, $1.0 \mathrm{ml}$ Tween $80,1.0$ Dextrose, $0.7 \mathrm{~g}$ L- cysteine, $0.5 \mathrm{~g}$ L-arginine, $2 \mathrm{~g}$ Disodium phosphate $\left(\mathrm{Na}_{2} \mathrm{HPO}_{4}\right), 4.0 \mathrm{~g}$ sodium acetate $\left(\mathrm{CH}_{3} \mathrm{COONa}\right), 0.1 \mathrm{~g}$ Magnesium sulfate $\left(\mathrm{MgSO}_{4} .7 \mathrm{H}_{2} \mathrm{O}\right), 0.5 \mathrm{~g}$ Manganese sulfate $\left(\mathrm{MnSO}_{4} \cdot 5 \mathrm{H}_{2} \mathrm{O}\right)$, in $1 \mathrm{ml}$ distilled filtered water (Table 1). Batches of $200 \mathrm{ml}$ containing different gums were sterilized at $1100 \mathrm{C}$ for $10 \mathrm{mins}$.

Table I Composition of modified basal medium for IL

\begin{tabular}{lll}
\hline Component & Supplier & Quantity (g/L) \\
\hline Proteose peptone \#3 & Remel & $2 \mathrm{~g}$ \\
Yeast extract & Neogen & 2.0 \\
Tween 80 & Fisher Scientific & $1.0 \mathrm{ml}$ \\
Dextrose & Fisher Scientific & 1.0 \\
L-cysteine & Fisher Scientific & 0.5 \\
L-arginine & Fisher Scientific & 0.5 \\
Disodium phosphate $\left(\mathrm{Na}_{2} \mathrm{HPO}_{4}\right)$ & Fisher Scientific & $2 \mathrm{~g}$ \\
Sodium acetate $\left(\mathrm{CH}_{3} \mathrm{COON}_{2}\right)$ & Fisher Scientific & $8.0 \mathrm{~g}$ \\
Magnesium sulphate $\left(\mathrm{MgSO}_{4} \cdot 7 \mathrm{H}_{2} \mathrm{O}\right)$ & Fisher Scientific & $0.1 \mathrm{~g}$ \\
Manganese sulphate $\left(\mathrm{MnSO}_{4} .5 \mathrm{H}_{2} \mathrm{O}\right)$ & Fisher Scientific & $0.05 \mathrm{~g}$ \\
\hline
\end{tabular}

Legend Basal medium composition was based on the composition of MRS with some modifications

\section{Growth study}

Sterilized basal medium samples were inoculated with $1 \%$ of inoculum at a final inoculum level of $3 \log \mathrm{CFU} / \mathrm{ml}$. Bacterial inoculum was prepared by taking aliquots $(1 \mathrm{~mL})$ from appropriate serial dilutions from each active culture and aseptically adding the aliquots to each $100 \mathrm{~mL}$ batch of $1 \%$ fat milk and basal medium samples. Samples without gum served as the control. Inoculated samples were serially diluted at a final inoculum level of 3 logs CFU/ $\mathrm{ml}$, plated on MRS agar, and incubated at $37^{\circ} \mathrm{C}$ for $48 \mathrm{~h}$ to determine initial bacterial counts. Inoculated samples were then incubated at $37^{\circ} \mathrm{C}$ for $12 \mathrm{~h}$. After incubation, samples were serially diluted and aliquots were placed on MRS agar to obtain final bacterial counts.

\section{Data and statistical analysis}

Each experiment was conducted three times in a randomized block design. The mean values and standard deviations were calculated from the duplicate tested samples. R-Project for Statistical Computing version R-2.15.2 (http://www.r-project.org) was used to determine significant differences between the effect of different gums on the growth of
Lactobacillus strains in milk and medium using one- way ANOVA (analysis of variance) at a significance level of $p<0.05$.

\section{Results}

\section{Growth of Lactobacillus strains}

A modified basal medium was prepared using basic components (Table 1) and nine different gums PE, CA, MC, PC, LB, GU, GL, IN and XA (Table 1) to study the effect of selected gums on the growth of Lactobacillus strains. The growth of Lactobacillus strains in the medium was compared to the growth in one percent fat fluid milk containing selected gums. Figures 1-3 shows the effect of gums on three Lactobacillus strains in laboratory media and in milk during 12 hours of incubation at $37^{\circ} \mathrm{C}$.

The population of the L.rhamnosus GGB101 strain was significantly $(p<0.05)$ higher in milk compared to medium in the presence of all tested gums (Figure 1). However, the highest bacterial population was observed in milk containing xanthan at $8.81 \pm 0.01 \log \mathrm{CFU} / \mathrm{mL}$, which was followed by milk sample containing carrageenan $(8.18 \pm 0.03 \mathrm{log}$ $\mathrm{CFU} / \mathrm{mL}$ ). The addition of guar-locust bean-carrageenan resulted in 
the least growth of L. rhamnosus GG B101 $(8.03 \pm 0.01 \log \mathrm{CFU} / \mathrm{mL})$ in milk. Similarly, the addition of xanthan led to significantly $(p<0.05)$ higher growth $(7.65 \pm 0.000 .04 \log \mathrm{CFU} / \mathrm{mL})$ of L. rhamnosus $\mathrm{GG}$ B101 in the medium, with locust bean inducing the least growth
(6.89 $\pm 0.06 \log \mathrm{CFU} / \mathrm{mL})$. However, the population of $L$. rhamnosus GG B101 was $1.16 \operatorname{logs} \mathrm{CFU} / \mathrm{mL}$ higher in milk than in media in the presence of xanthan (Figure 1).

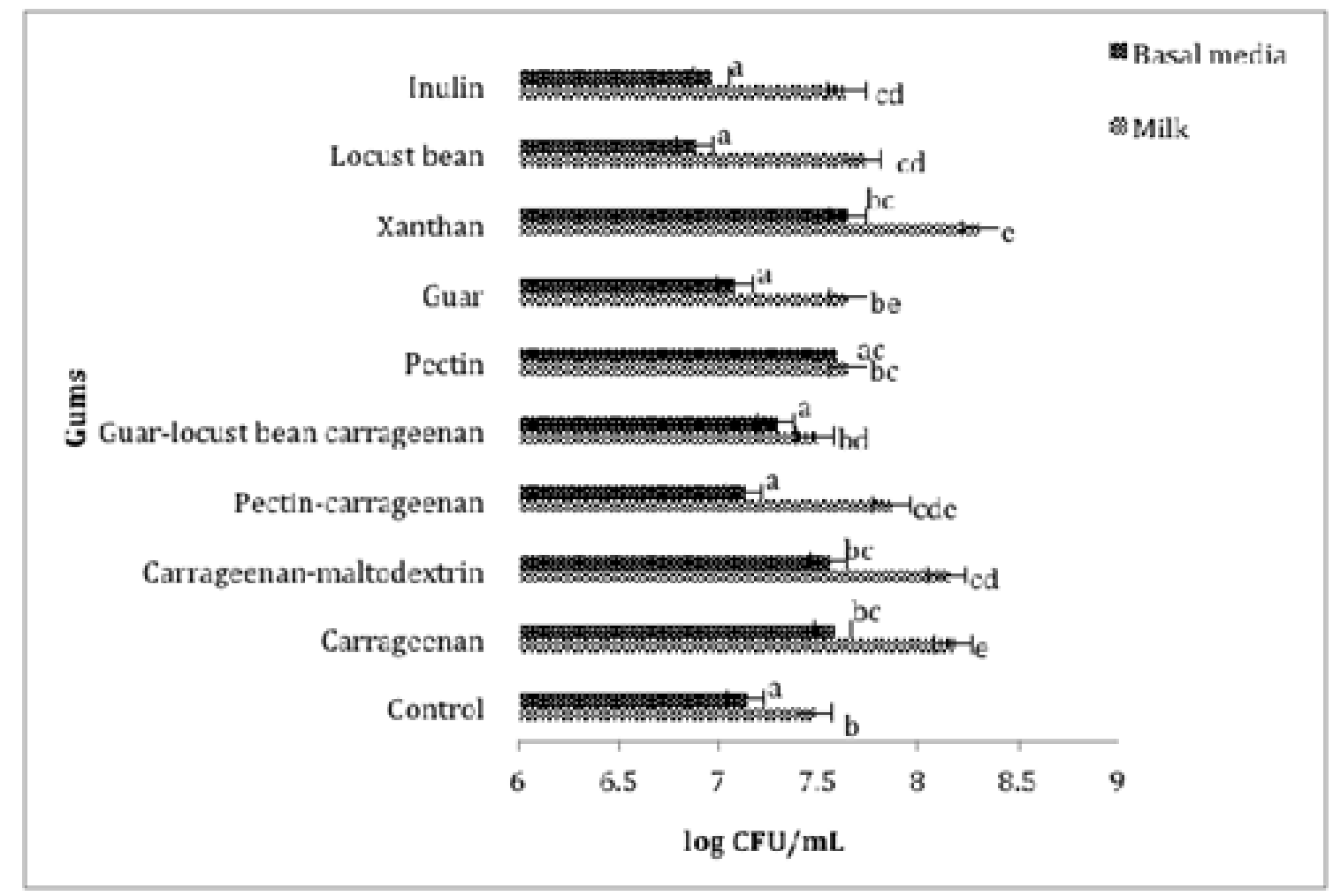

Figure I Comparing the effect of gums on the growth of L. rhamnosus GG BIOI in basal media and milk during I 2 hours of incubation at $37^{\circ} \mathrm{C}$.

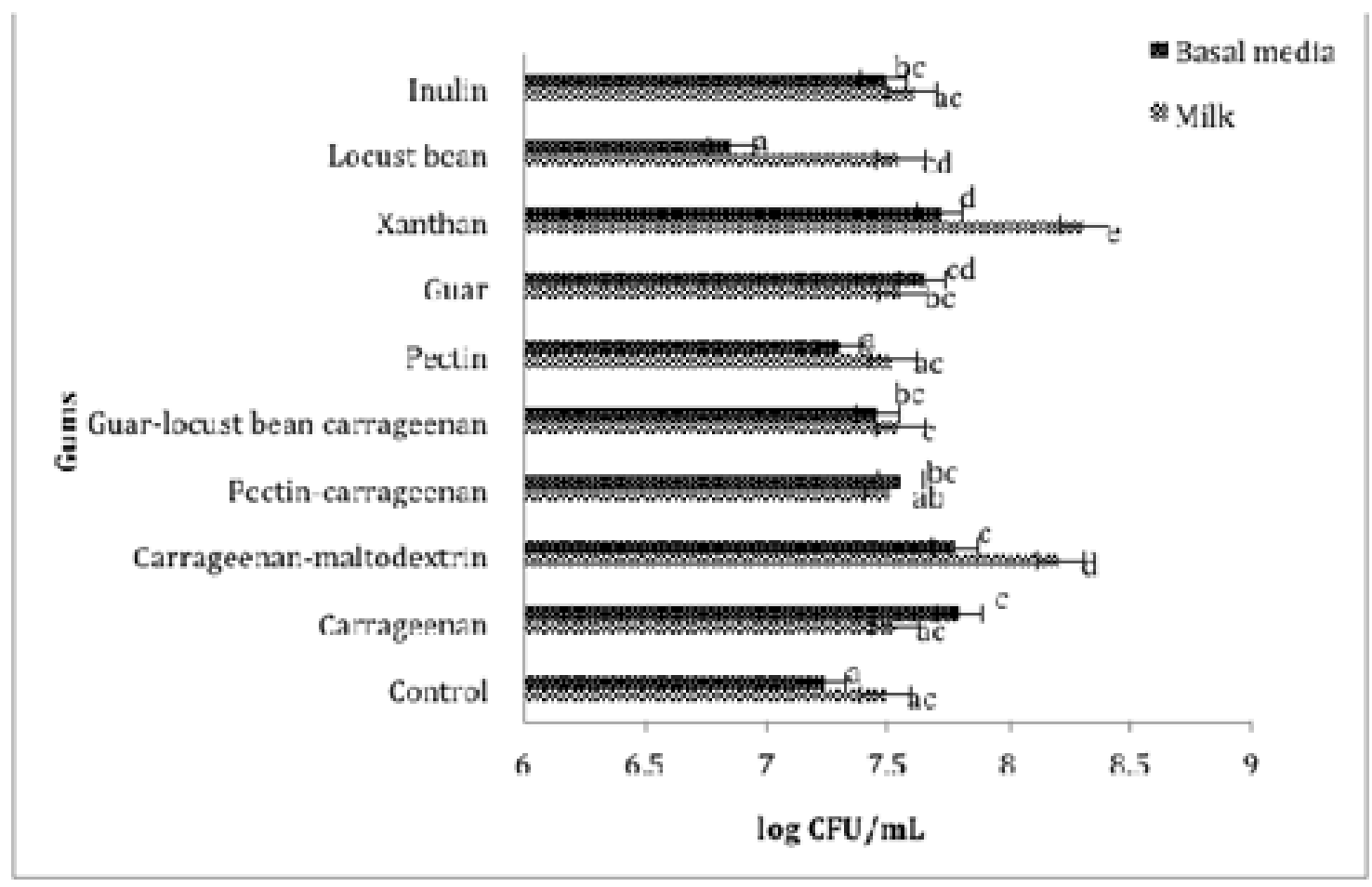

Figure 2 Comparing the effect of gums on the growth of L. rhamnosus GG BI03in basal media and milk during I 2 hours of incubation at $37^{\circ} \mathrm{C}$. 




Figure 3 Comparing the effect of gums on the growth of L. reuteri DSM200I6 in basal media and milk during I 2 hours of incubation at $37^{\circ} \mathrm{C}$.

Significantly $(p<0.05)$ higher population of L. rhamnosus GG B103 was observed in milk compared to medium samples in the presence of all tested gums, except in the milk sample containing guar, pectin-carrageenan, and carrageenan where a slightly higher population of L. rhamnosus GG103 was found (Figure 2). The addition of xanthan led to the highest population of L. rhamnosus GG B103 $(8.32 \pm 0.01 \log \mathrm{CFU} / \mathrm{mL})$ in milk during the incubation period. Compared to the control, the population of L. rhamnosus GG B103 was significantly $(p<0.05)$ lower in the presence of pectin and locust beans in the medium, indicating an inhibiting effect of these gums on the growth of $L$. rhamnosus GG B103. The addition of tested gums led to a positive effect on the population of L. rhamnosus GG B103 in all milk samples compared to medium (Figure 2).

The presence of carrageenan-maltodextrin resulted in the highest population of L.reuteri DSM20016 $(8.3 \pm 0.23 \log \mathrm{CFU} / \mathrm{mL})$ in modified basal media during the incubation period (Figure 3). It was notable that carrageenan, carrageenan-maltodextrin, guar, and inulin promoted higher growth in the media compared to milk and also promoted higher growth in treatments than in the control. In contrast, gums (pectin-carrageenan, pectin, xanthan, guar-locust bean-carrageen and carrageenan) that led to a higher population of L.reuteri DSM20016 in treated samples than in control samples also enhanced bacterial growth in milk. This would indicate that the metabolic activity of Lactobacillus strains was affected directly by gums and indirectly by the medium of growth. This effect could be due to the interaction between the gums and the strains. L. rhamnosus GG that grew best in milk compared to the L. reuteri strain.

Carrageenan, carrageenan-maltodextrin, guar, inulin and locust bean enhanced the growth L. reuteri DSM20016 in media. However, the addition of pectin-carrageenan, pectin, guar-locust beancarrageenan and xanthan resulted in a slightly higher population of L. reuteri DSM20016 in milk (Figure 3). The presence of carrageenan-maltodextrin led to the highest population of $L$. reuteri DSM20016 $(8.3 \pm 0.23 \log \mathrm{CFU} / \mathrm{mL})$ in media. It was also observed that carrageenan, carrageenan-maltodextrin, guar, inulin and locust bean which induced higher growth in the treatment group than in the control, also promoted higher growth in media compared to milk. Similarly, gums (pectin-carrageenan, pectin, xanthan, guar-locust bean-carrageen and carrageenan) that led to higher populations of strains in treated samples than in control samples also improved the growth of L. reuteri DSM20016 in media compared to milk. Xanthan significantly $(p<0.05)$ stimulated the highest growth of $L$. rhamnosus GG strains in both milk and media whereas the addition of xanthan resulted in aslight inhibition in the L. reuteri strain in both milk and media. The presence of carrageenan-maltodextrin and carrageenan significantly $(p<0.05)$ promoted the growth of all tested Lactobacillus strains in both media and milk.

\section{Discussion}

The results from this study demonstrated that gums could promote the growth of Lactobacillus strains. These results support previous findings ${ }^{12,14}$ that showed carrageenan-maltodextrin could promote growth and viability of Lactobacillus strains.

According to Su et al., ${ }^{4}$ the addition of certain prebiotics such as fructose oligosaccharides (FOS) and soybean oligosaccharides (SOS) at $1.5 \%$ enhanced the growth of lactobacillus in a basal medium at about $1 \log \mathrm{CFU} / \mathrm{mL}$. Buttermilk and whey supplemented with yeast extract also improved the growth of Lactobacillus strains, although the growth was lower compared to MRS. ${ }^{15}$ The ability of gums to impact the growth of Lactobacillus could be due to the interaction between gums and the tested Lactobacillus strains as the metabolic activity of Lactobacillus strains tend to respond to the composition of the medium 
of growth. ${ }^{16}$ Additionally, the structure and composition of gums could be a key factor in the enhancement of bacterial growth. Gums vary in chemical composition and carbon chains which impacts on their digestibility and availability to bacteria. According to HernandezHernandez et al., ${ }^{10}$ the length of carbon chains in carbohydrates such as galactooligosaccharides affects digestibility. The longer the carbon chains as in xanthan, the slower the digestibility, which subsequently impacts the growth of lactobacilli. We would therefore expect to find the same trend in the digestibility of selected gums due to their composition and structure as well as carbon chain length with their consequent effect on growth and viability. ${ }^{10}$ Additionally, gums added carbon and energy sources for the hetero fermentative metabolic activity by $L$. reuteri and $L$. rhamnosus strains leading to enhance growth in the milk and media containing gums. ${ }^{16}$

\section{Conclusion}

Our results indicated that the tested gums could enhance the growth of Lactobacillus strains in milk compared to media as the addition of gums significantly improved the population of Lactobacillus strains in milk. In addition, carrageenan-maltodextrin and carrageenan showed enhanced growth of Lactobacillus strains in both milk and media. Xanthan stimulated the highest growth of L. rhamnosus GG strains but led to a slight inhibition in the growth $L$. reuteri strain in both milk and media.

\section{Acknowledgements}

This study was conducted at North Carolina Agricultural and Technical State University and supported by a grant from the USDA.

\section{Conflict of interest}

Author declares that there is no conflict of interest.

\section{References}

1. FAO-WHO. Guidelines for the evaluation of prebiotics in Food. Report of a joint FAO/WHP working group on drafting guidelines for evaluation of probiotics in food. London, Ontario, Canada; 2002.

2. Gibson GR. Fiber and effects on probiotics (the prebiotic concept). Clinical Nutrition Supplements Journal. 2004;1(2):25-31.

3. Hekmat S, Soltani H, Reid G. Growth and survival of Lactobacillus reuteri RC-14 and Lactobacillus rhamnosus GR-1 in yogurt for use as a functional food. Innovative Food Science \& amp; Emerging Technologies. 2009;10(2):293-296.

4. Maganha LC, Rosim RE, Corassin $\mathrm{CH}$, et al. Viability of probiotic bacteria in fermented skim milk produced with different levels of milk powder and sugar. International Journal of Dairy Technology. 2014;67(1):89-94.
5. Quigley EM. Prebiotics and probiotics; modifying and mining the microbiota. Pharmacol Res. 2010;61(3):213-218.

6. Siok-Koon Y, Min-Tze L. Effect of prebiotics on viability and growth characteristics of probiotics in soymilk. J Sci Food Agric. 2010;90(2):267-275.

7. Gibson GR, Probert HM, Loo JV, et al. Dietary modulation of the human colonic microbiota: updating the concept of prebiotics. Nutr Res Rev. 2004;17(2):259-275.

8. Zárate G, Pérez CA. Influence of lactose and lactate on growth and $\beta$ galactosidase activity of Potential probiotic Propionibacterium acidipropionici. Anaerobe. 2012;18(1):25-30.

9. Corona-Hernandez IR, Álvarez-Parrilla E, Lizardi-Mendoza J, et al. Structural Stability and Viability of Microencapsulated Probiotic Bacteria. A Review Comprehensive Reviews in Food Science and Food Safety. 2013;12(6):614-628.

10. Hernandez-Hernandez O, Muthaiyan A, Moreno FJ, et al. Effect of prebiotic carbohydrates on the growth and tolerance of Lactobacillus. Food Microbiol. 2012;30(2):355-361.

11. Ibrahim SA, O'Sullivan DJ. Use of Chemical Mutagenesis for the Isolation of Food Grade $\beta$ Galactosidase Overproducing Mutants of Bifidobacteria, Lactobacilli and Streptococcus thermophilus. J Dairy Sci. 2000;83(5):923-930.

12. Karlton-Senaye BK, Ibrahim SA. Impact of gums on the growth of probiotics Agro Food Industries. Functional food Nutraceutical. $2013 ; 24: 10-14$

13. Heydari S, Mortazavian AM, Ehsani MR, et al. Biochemial, Microbiological and sensory characteristics of probiotic yogurt containing various prebiotic compounds. Italian Journal of Food Science. 2011;23(2):153.

14. Karlton-Senaye BD, Tahergorabi R, Giddings V, et al. Effect of gums on the viability and $\beta$-galactosidase activity of Lactobacillus spp. in milk drink. International Journal of Food Science and Technology. 2014;50(1):32-40.

15. Burns P, Vinderola G, Molinari F, et al. Suitability of whey and buttermilk for the growth And frozen storage of probiotic lactobacilli. International Journal of Dairy Technology. 2008;61(2):156-164.

16. De Souza RP, Perego P, De Oliveira MN, et al. Effect of inulin on the growth and metabolism Of a probiotic strain of Lactobacillus rhamnosus in co-culture with Streptococcus thermophilus LWT. Food Science and Technology Journal. 2012;47(2):358-363. 